\title{
A cost-utility analysis of small versus large bite sutures in the closure of midline laparotomies in the UK NHS
}

Shyam Gokani, Karl Elmqvist, Osman El-Koubani, Javier Ash, Sudeep Biswas, Maxime Rigaudy

Imperial College Business School, London, UK

Correspondence to shyamg@live.co.uk

cite as: Gokani S, Elmqvist K, El-Koubani O, Ash J, Biswas S \& Rigaudy M. (2020) A cost-utility analysis of small versus large bite sutures in the closure of midline laparotomies in the UK NHS. The Physician $6(1) c 8$ DOI: 10.38192/1.6.1.c8

\section{Background:}

This study aimed to perform an economic evaluation of small bite sutures versus large bite sutures in the closure of midline laparotomies in the United Kingdom National Health Service (NHS).

\section{Methods:}

A cost-utility analysis was conducted using data from a systematic literature review. Large bite sutures placed $10 \mathrm{~mm}$ from the wound edge were compared to small bite sutures 3-6 $\mathrm{mm}$ from the wound edge. The analysis used a 3-year time horizon in order to take into account complications including incisional hernias and surgical site infections (SSIS). Cost and benefit data were considered from the perspective of the NHS. A two-way sensitivity analysis was conducted to assess the impact of a variation in the clinical effectiveness of small bite sutures.

\section{Results:}

The incremental cost-effectiveness ratio was calculated to be $-£ 482.61$ per quality-adjusted life year (QALY) using the proposed small bite suture technique, indicating a cost saving to the NHS. Sensitivity analysis demonstrated that small bites are a cost-neutral technique provided that the cost of using small bites is less than $£ 98$ per patient. Small bites cost less than $f 20,000 / Q A L Y$ when they reduce either the rate of SSIs by more than $15 \%$ or the rate of hernias by more than $3.4 \%$.

\section{Conclusion}

This study proposes that small bite sutures should become the mainstay suturing technique in the closure of midline laparotomies, replacing large bite sutures, which dominate current practice. The financial savings accompanied by the decrease in SSI rates and herniation warrant the use of this new technique. 
The sensitivity analysis demonstrates that findings hold true for a wide range of levels of clinical effectiveness for small bites. 